\title{
Longer-term effects of coffee on glucose and lipid metabolism
}

\author{
T.M. Robertson ${ }^{1}$, M.N. Clifford ${ }^{1}$, S. Penson ${ }^{2}$ and M.D. Robertson ${ }^{1}$ \\ ${ }^{1}$ University of Surrey, Guildford, GU2 $7 X H$ and ${ }^{2}$ Campden BRI, Chipping Campden, GL55 6LD
}

There is much epidemiological evidence demonstrating an association between coffee drinking and reduced incidence of Type 2 Diabetes ${ }^{(1)}$, however there have been few longer term interventional studies, the majority of which have recruited habitual coffee drinkers $^{(2)}$. Habitual coffee drinkers will have already gained any potential long-term benefits of coffee drinking and so the results of such intervention trials may be misleading. Additionally, previous studies may be confounded by inter-individual variation in caffeine metabolism. The rs762551 single nucleotide polymorphism (SNP) in the CYP1A2 gene has been demonstrated to influence caffeine metabolism, with carriers of the $\mathrm{C}$ allele being characterised as having a "slow" metaboliser phenotype. An association between coffee drinking and impaired fasting glucose has been observed, with a stronger association reported in the "slow" metaboliser phenotype ${ }^{(3)}$, however, to date, no coffee-intervention studies have genotyped their participants for this SNP.

This parallel-arm intervention investigated the effects of 12 weeks of coffee drinking on measures of glucose and lipid metabolism in coffee-naïve individuals, with secondary analysis by CYP1A2 genotype. Participants in the coffee group $(\mathrm{n}=19)$ consumed 4 cups/day instant coffee for 12 weeks, whilst those in the control group $(\mathrm{n}=8)$ remained coffee/caffeine free. Fasted and $2 \mathrm{~h}$ postprandial venous blood samples were taken at the start and end of the intervention.
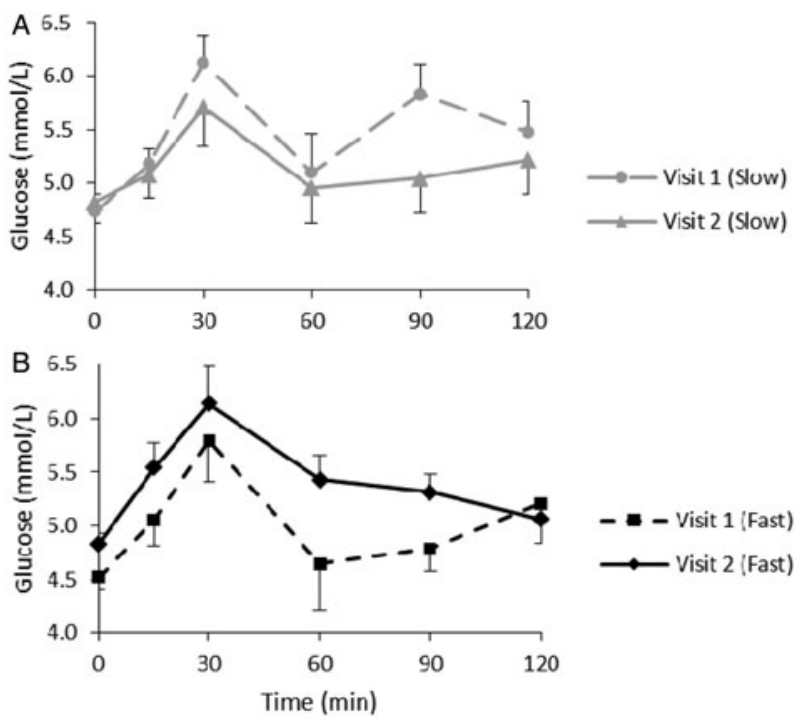

Fig. 1. Postprandial glucose response by visit.

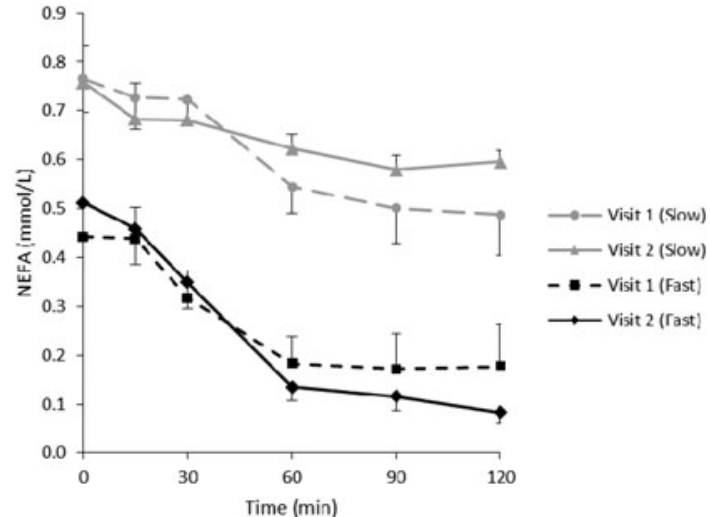

Fig. 2. Postprandial NEFA response by visit.

There were no significant differences between coffee and control groups in their response to the intervention for any measure, however, secondary analysis of the coffee group by genotype revealed several differences. Those of a "slow" caffeine metaboliser phenotype $(n=9)$ displayed higher postprandial glucose and higher fasted and postprandial NEFA at baseline $(\mathrm{p}<0.05)$ than those with the "fast" phenotype $(n=10)$. A visit*phenotype interaction was observed for the postprandial glucose response $(p<0.05)$, with the "fast" phenotype displaying an increased glucose response following the coffee intervention (Fig. 1B) and the "slow" phenotype displaying a reduced response (Fig. 1A). A visit*phenotype*time interaction was observed for the postprandial NEFA response $(\mathrm{p}<0 \cdot 05)$, with the "fast" phenotype displaying greater NEFA suppression following the intervention and less NEFA suppression observed in the "slow" phenotype (Fig. 2).

These observed differences between "slow" and "fast" caffeine metaboliser phenotypes warrant further investigation but indicate there should be no one-size-fits-all recommendation with regard to coffee drinking and Type 2 Diabetes risk.

1. Ding M, Bhupathiraju SN, Chen M, et al. (2014). Diabetes Care. 37, 569-86.

2. Kempf K, Herder C, Erlund I, et al. (2010). Am J Clin Nutr. 91, 950-7.

3. Palatini P, Benetti E, Mos L, et al. (2015). Eur J Epidemiol. 30, 209-17. 